\title{
Schema Theory, Construction-integration reading model and Reading
}

\section{Pedagogy}

\author{
Wenqi Xiao
}

B511-2, Kunluan Building, International College, Xiamen University (Xiang-an Campus), Xiang-an south road, Xiang-an District, Xiamen, Fujian, China

Key words: reading comprehension, schema theory, construction-integration reading model

\begin{abstract}
Reading comprehension involving complicated cognitive process deservesintensive research focus. The development of schema theory gives many insights into understanding the process in that it explains how background knowledge is activated to facilitate comprehension. With the major contributions, schema theory also arises discussions and arguments. Another reading model from construction-integration theory emphasizing on constructing text-based knowledge from text input offers a different perspective. The review of the two reading models is a humble attempt to discuss comprehension process and some pedagogical implications are drawn. It is suggested that prior knowledge should be enhanced in reading activities and lower-processing reading skills also needs instructing for better comprehension.
\end{abstract}

\section{Introduction}

Reading comprehension is a complex cognitive process.Nuttall[1] describes reading comprehension as obtaining the writer's intended meaning through the text. The schema theory providing explanations of how knowledge is represented, used and inferred gives insights of the cognitive process. This theory emphasizing on the role of background knowledge in facilitating reading comprehension has prompted the development of research and instruction of L2 reading. On the other hand, the construction-integration theory proposed by Kintsch[2] provides an alternative view of interpreting reading comprehension. Reading comprehension begins with generating text-based knowledge and by integrating the structured text-based knowledge with the reader's general knowledge, a coherent mental representation of the text is constructed. Even though the two theories have different perspectives, they are both conducive in understanding reading comprehension.

This essay will first introduce the development and arguments of schema theory. Then discussion will focus on construction-integration theory and its applications in explaining L2 readers' reading problems and habits. Finally, implications will be presented.

\section{Schema theory}

\section{Origin and development.}

The notion of schema was proposed by Barlett[3] who perceived schema as organization of information or past experience stored in memory. Schema, according to Barlett, is used to retrieve and recall the information [3].

With the emergence of computer science and artificial intelligence,the schema theory is well developed as a theoretical framework in describing organization and knowledge in mind. A large 
host of empirical studies haven been carried by adopting the theory in explaining cognitive process. Schema theory perceives human memory as organized and structured schemata. Thisprovides insights in understanding cognitive process including memorizing, retrieving, inferring, and comprehension [4].

\section{Schema theory model of reading.}

A reading model interpreting reading as an interactive process between reader's background knowledge and the text is developed based on the schema theory[5]. The background knowledge is the schema derived from reader's past experience and contributes a lot to comprehension. The major connotations of the theory are presented as follows.

\section{Pre-existing prior knowledge.}

There are pre-existing prior knowledge or background knowledge in reader's mind, and these background knowledge are prerequisites of new knowledge. New knowledge is acquired when there is relevant pre-existing knowledge [6].

\section{Selection.}

Of all the given information, only part of the input that is relevant with pre-existing knowledge is selected and comprehension is a process of matching the relevant information from the text with pre-existing background knowledge [5]. In other words, acquisition and comprehension is based on pre-existing and relevant background knowledge.

\section{Reader-driven process.}

Readers from different cultures and societies relate to the text in different ways and approach meanings on the basis of their cultural and social background [7].

\section{Discussions.}

1. New knowledge derives from pre-existing prior knowledge implies the importance of activating current schemata but may neglect the importance of creating new schemata. Although it has been pointed that schema can be modified, increased [8], most experimental research emphasized on activating pre-existing schemata [4]. If the reader does not obtain the schema that can map against the information, comprehension cannot take place.

2.Schema theory background knowledge has determinant role reading comprehension. However, proficient L2 readers are those who can integrate the use of background knowledge (top-down approach)with sematic and synaptic aspects of the text (bottom-up approach).

\section{Construction-integration reading model}

With research on human memory and recall, Kintsch and his colleagues proposed the discourse comprehension model. With more elaborations and modification, Kintsch[2]developed the construction and integration theory as model of text comprehension. Comprehension relies on the knowledge from the text, rather than the pre-existing knowledge and there aretwo processes: construction and integration.

In the construction process, smallest units of ideas or propositions are generated from words, sentences, or context. Then knowledge net that isboth relevant and irrelevant with the propositions will be activated. With continuous inference and additional propositions, the knowledge net will be 
refined. Propositions representing local meaning will be organized as microstructure and higher-level relationships will form a macrostructure.Integration takes place as the knowledge net is constructed. It is a modified process involving text networks from semantic level, syntactic level, discourse level and integration occurs in a repeated circle. As the new network of text meaning is formed, it is integrated with the previous circle from the working memory. Integration circle operates until all incongruities are diminished and coherent interpretation is formed.

The core idea of the theory is that comprehension is generated from the textual information instead of knowledge outside the text. Compared with a top-down approach, from construction and integration perspective, knowledge is constructed from all textual levels and refined through reading process.

\section{Applications and discussions.}

\section{L2 reader's reading speed.}

Research has found that compared to reading in first language, L 2 readers are slower when reading in second language [9]. It is also discovered that L2 readers read in a repeated and slower manner than L1 readers [10]. Schema theory explains the phenomenon by suggesting that L2 readers are less capable of adopting higher-level process strategies. However, this explanation is not consistent with research evidence. It is proved that L2 readers are able to utilize high-order strategies and adopt more extensively than L1 readers [11]. From the perspective of construction-integration, it is the efficiency in construction and integration process that slows L2 readers down. In the construction process readers who are less proficient in knowledge levels including lexical, syntactic, discourse proceed text with more time. Without the formation of text base, it is hard to integrate meanings and constructing coherent mental representation. Thus, L2 readers may read repeatedly and slowly.

\section{Text processing skills.}

It is suggested that L2 readers and L1 readers share similar text processing skills. Both L2 and L1 readers approach the sentence by breaking down into small units such as phrases, clauses on the basis of semantic and syntactic knowledge, and integrate the linguistic units into large discourse. However, due to less adequate sociocultural background information, L2 readers relies more on text information and linguistic proficiency to obtain meanings [12].

\section{Conclusions}

Under complex cognitive process, it is not easy to accurately identify the whole reading process and comprehension. Without doubts, schema theory has given insights in understanding the process and prompted research development L2 reading. When applying to reading comprehension, this theory employs a more top-down approachand emphasizes the contributing role of background knowledge to reading comprehension. On the other side, the construction-integration theoryfocusing on generating knowledge on the basis of text itself provides an alternative view in which semantic and syntactic levels of text input constructs text base and integrates to form coherent mental representation. This theory is initially applied in L1 reading and more research should be carried in the context of L2 reading. Although the two theories are distinct, arguments and discussions on the two theoriesin fact give importantimplications in L2 reading instructions. 


\section{Create and enhance background knowledge.}

As mentioned in schema theory, it is not deniable that pre-existing background knowledge facilitates comprehension. However, creating background knowledge should be stressed to enhance schemata, instead of holding a static view on prior knowledge. In terms of reading instruction, providing learners with background knowledge of the text helps them activate pre-existing knowledge or create new background knowledge. As a result, it can not only prompt reading comprehension but also increase students' schemata.

\section{Learner discussions and sharing.}

With different sociocultural background, learners' comprehension of the text varies and embodies individual interpretation. This ...implies the value of learner discussions after reading comprehension. Teachers should give students opportunities to share their understanding and reasons. By doing so, individual feels being respected but more important students learn from each other to obtain a greater range of schema.

\section{Emphasis on lower-level processing skills.}

Slower reading speed and repeated reading may not come from lacking of top-down strategies, especially for adolescent learners. Teachers should consider this reading problem may derive from deficiency in lower-level processing skills such as lexical knowledge, syntactic knowledge. With the understanding of L2 readers' reliance on linguistic units, teachers should help cultivate students' capability in words recognition and inference, syntactic analysis and clauses identification, in order to construct text-based knowledge.

\section{References}

[1] C. Nuttall, Teaching Reading Skills in a Foreign Language, London, Heinemann Educational, 1982.

[2] W.Kintsch, The role of knowledge in discourse comprehension: A construction-integration model. Psychological Review, 92(1988), 163-182.

[3] F. C. Bartlett, Remembering. Cambridge: Cambridge University Press, 1932.

[4] H. Nassaji, Schema theory and knowledge-based processes in second language reading comprehension: a need for alternative perspectives, Language Learning, 52(2002), 439-481.

[5] P.L.Carrell, J.C.Eisterhold, Schema theory and ESL reading pedagogy, TESOL Quarterly, 17(1983), 553-573.

[6] J.W. Alba, L, Hasher, Is memory schematic? Psychological Bulletin, 93(1983), 203-231.

[7] S.Khanam, S.H. Zahid, S, Mondol, The role of schema for effective EFL reading comprehension, ASA University Review, 8(2014), 84-93.

[8] J.R. Anderson, R. Paulson, Representation and retention of verbatim information, Journal of Verbal learning and behavior, 16(1977), 439-451.

[9] M, Mack. A study of semantic and syntactic processing in monolinguals and fluent bilinguals.Journal of Psychological Research, 15(1986), 463-489.

[10] M, Clarke. The short circuit hypothesis of ESL reading-Or when language competence interferes with reading performance.Modern Language Journal, 64(1980), 203-209.

[11] Y. Horiba, Reader control in reading: Effects of language competence, text type and task. Discourse Processes, 29(2000), 223-267.

[12] Y. Horiba, Comprehension processes in L2 reading: Language competence, textual coherence, 
and inferences. Studies in Second Language Acquisition, 18(1996), 403-432. 